DOI: http://dx.doi.org/10.7551/978-0-262-33027-5-ch028

\title{
Environmental Factors and the Emergence of Cultural - Technical Innovations
}

\author{
Peter Andras ${ }^{1}$ \\ ${ }^{1}$ School of Computing and Mathematics, Keele University, UK \\ p.andras@keele.ac.uk
}

\begin{abstract}
Environmental factors that determine ecological niches, for example natural boundaries formed by mountains, rivers, deserts, contribute to the speciation among animals. Similar factors have been proposed to be important for the emergence of cultural and technical innovations in human populations in the pre-state stages of societies. Here we describe a social simulation aimed to investigate this issue. The simulation uses two environmental features, mountain ridges and the fertility of the land. The results show that indeed these environmental factors matter for the emergence of successful innovative populations. The defenses provided by mountain ridges facilitate the emergence of many populations with moderately successful innovations. The fertile lands are where the populations with the most successful innovations emerge, however in most cases these populations can trace their origins to innovative populations emerging under the defense of mountain ridges. This simulation study provides experimental support for the relatively speculative theories about the importance of environmental factors for the emergence of cultural and technical innovations.
\end{abstract}

\section{Introduction}

The emergence of cultural - technical innovations in human populations determined the dynamics of locally and globally dominant cultures across history (Bouckaert et al, 2012; Chiaroni et al, 2008; Diamond and Bellwood, 2003; Mellars, 2006). The factors that promote the emergence of such innovations are not well established and there are several theories that aim to explain these events (Diamond, 1997; Diamond and Bellwood, 2003; Fukuyama, 2014). In general such innovation give a competitive advantage to the populations that adopt them in the context of their physical and social environment, making these populations grow faster than others or have better chance of winning in battles consider for example the emergence of agriculture, the domestication of cows and horses, the flexible use of fast light cavalry by migratory populations in Europe and Asia in the middle ages, or the introduction of cannons in the late middle ages in Europe, Asia and North Africa (Diamond and Bellwood, 2003; Fukuyama, 2014; Mellars, 2006).

Considering early human populations some areas of the Earth stand out as relatively frequent sources of major waves of culturally - technically dominant populations and others stand out as the most culturally diverse areas. For example, the area between the Altay Mountains and the Fergana Valley in Central Asia has been the source of several waves of radiation of culturally - technologically dominant populations that went on to control, rule and dominate large parts of the world - e.g. Mongols, Turkic populations that established empires in Western and South Asia and regularly threatened or even conquered China (Fukuyama, 2014). Examples of high linguistic and cultural diversity include the highlands of Papua New Guinea (Reesink et al, 2009) and the Caucasus (Bulayeva et al, 2003).

In the case of animals and plants speciation is driven by the emergence of functional innovations that give a competitive fitness advantage to individuals having these innovative features in the context of some ecological niche (Barraclough et al, 1998; Warren et al, 2008). Examples include shrinking of size in island populations of animals, ability to access and digest new sources of food, or the emergence of the ability to glide and fly. In these cases the physical boundaries (e.g. large mountains or rivers, deserts) and features of the environment (e.g. humidity, aridity, temperature, light conditions) often play an important role in the definition of the environmental niche where the functional innovations may convey fitness advantage (Barraclough et al, 1998).

It has been proposed that environmental factors, such as mountain and rivers barriers, land fertility, presence of disease vectors (e.g. mosquitoes, flies), matter for the likelihood of emergence of cultural - technical innovations in human populations (Fukuyama, 2014). For example, many relatively isolated and hard-to-access valleys in highland areas may facilitate the maintenance of many separately developing populations providing them sufficient protection from neighbors to develop and perfect potentially advantageous cultural and technical innovations. Or, proximity to fertile lowland areas may facilitate the expansion and the development of further advantageous innovations in growing populations emerging from protected valleys, which already have some cultural -technical advantage over other neighboring populations.

However, these theories and proposals rely to a good extent on speculations constrained by the available historical and archaeological evidence. Naturally, there is relatively little objective evidence to confirm these theories. One possible approach for gathering more supporting evidence is to build simplified simulations of evolution and spreading of human populations including the simulation of emergence of cultural - technical innovations and test assumptions about the roles of environmental factors through analysis of the outcome of these simulations.

Here we describe a simulation of evolution and spreading of human populations, including the simulation of natural boundaries as mountain ridges and the variability of the land 
fertility as environmental factors with expected impact on the emergence of cultural - technical innovations. The simulated human populations are enabled to generate cultural - technical innovations that change their fitness for growth. We analyzed the impact of the considered two environmental factors on the emergence of these innovations by considering populations that are successful in maintaining themselves over considerable simulated time and in achieving sufficiently large relative size among the simulated populations.

Our results show that the proximity of mountain ridges that protect from conquest by neighbors and the low land fertility of the area correlate with innovations that confer large positive changes in the competitive fitness of the simulated human populations. The results also show that the most dominant populations originate from fertile lowlands, however in all cases the roots of these populations go back to places close to mountain ridges. Overall, the simulation results confirm the importance of the considered environmental factors for the emergence of cultural technical innovations.

The rest of the paper is organized as follows. First we review the related research. Then we describe in detail the simulation of evolution of human populations constrained by environmental factors. Then we present the simulation results and their analysis. The paper is closed by a discussion and conclusions section.

\section{Related Works}

The emergence and evolution of animal and plant species and their spreading across areas of the Earth has been researched for long time. In general, ecological niches provide the setting for the emergence of new species and the species spreads territorially and to other ecological niches by outcompeting other species resident in these spaces (Barraclough et al, 1998; Warren et al, 2008). The role of environmental barriers, such as mountain ridges, deserts, sea, large rivers and lakes and vast and dense forests, has been studied in the context of ecological niche formation (Barraclough et al, 1998; Warren et al, 2008).

The evolution of human populations and the emergence of cultural or technical innovations that give an advantage to a population in comparison with neighboring populations have been considered as an intriguing question by many researchers (Diamond, 1997; Fukuyama, 2014). There is increasing volume of data, e.g. archaeological data, population genetics data, linguistic data, that can be used to support theoretical proposals in this area of research (Bouckaert et al, 2012; Chiaroni et al, 2008; Der Sarkissian et al, 2013; Diamond and Bellwood, 2003; Reesink et al, 2009). However, the limited nature of all these data means that often it is difficult to decide about the validity of these theoretical proposals.

In general it is assumed by many researchers that environmental factors such as well separated highland valleys, or alluvial valleys of large rivers, or lack or abundance of fertile land, contribute to the chances of human populations inhabiting such areas to survive, become dominant in a larger area, and to develop successful cultural or technological innovations (Diamond and Bellwood, 2003; Fukuyama, 2014; Mellars, 2006). For example, protected highland valleys may allow the co-existence of many culturally / technologically different human populations, which may try out many potentially successful innovations. Some of these innovations may confer sufficient competitive advantage to the inventor population to conquer other populations over extended areas that offer less natural protection if such areas are available not too far from the original location of the inventor population. Some argue about the importance of alluvial valleys or fertile land for the expansion of the population, which is required as a pre-requisite for the later conquest of other populations and areas (Fukuyama, 2014).

The modeling of spreading of populations has a well established mathematical theory rooted in the study of reaction - diffusion equations (Garcia-Ramos and Rodrigues, 2001; Petrovskii et al, 2002). The general equation of this theory describes the spatio-temporal diffusion of the population combined with a reaction term that represents the impact of external factors, for example the local growth of the population as a function of available resources and efficiency of use of these resources. The equation is as follows:

$$
\partial u(x, t) / \partial t=\nabla(D(u, x) \cdot \nabla u(x, t))+F(u, x, t)
$$

where $u(x, t)$ is the size of the population at spatial position $x$ and at time $t$. The term $D(u, x)$ describes the diffusion of the population at location $x$, and $F(u, x, t)$ is the reaction term that describes the change in the size of the population at spatial location $x$ and at time $t$ due to the external factors. In general this equation cannot be solved, but solutions of particular cases can be found. Another approach is to solve step-by-step a discretized version of the equation.

It has been shown that population dispersion described through equation (1) leads to wavefront propagation phenomena and Turing patterns of waves (Cheng et al., 2014; Jeltsch et al, 1997; Lambin et al, 1998). The wavelength of these patterns depends on the diffusivity of the population, the quicker is the diffusion the shorter is the wavelength of the patterns, and in general the wavelength is proportional to the square-root of the diffusivity value (Ouyang et al, 1995).

Simulation based modeling of population spreading follows from the discretized version of equation (1). Computer simulations have been developed to model particular cases of population spreading (e.g. spreading of certain invasive species) (Cheng et al, 2014; Garcia-Ramos and Rodrigues, 2001). The simulations often assume homogeneous diffusivity, i.e. the same diffusivity everywhere. The assumptions about the reaction term are based either on some reproduction and death dynamics (i.e. a combination of adding and subtracting a number of individuals on the basis of an equation describing this population size dynamics) or on interactions with one or more other species. The simulations of population spreading produce the expected Turing patterns of traveling waves (Cheng et al., 2014; Petrovskii et al, 2002). The Turing patterns are generated in a uniform manner, as the reaction terms usually apply uniformly across the whole simulated space. The patterns observed in the simulations are similar to the actual observation of spreading of species in cases where sufficient data is available (Jeltsch et al, 1997; Lambin et al, 1998). 
The spreading of innovations across populations has been studied for decades (Mahajan and Muller, 1979). These works focus on how innovations spread through adoption by populations and the results are similar to the spreading of infectious diseases (Delre et al, 2010; Macy and Willer, 2002). In this paper we do not consider the adoption of cultural and technical innovations by imitation across populations.

\section{Simulation of the Evolution of Human Populations}

The simulated world is a 2-dimensional grid of $100 \times 60$ spatial locations. Each location has a height and a harshness value. The height gives the altitude of the location and the harshness is the opposite of the land fertility. The height value is an integer between 0 and $g_{\max }=100$. The harshness value is a real value between 0 and 1 , larger value meaning harsher, less fertile, spatial location. The height values are set such that the locations arranged along lines have the same height value - these locations together form a simulated mountain ridge. Each simulated world has $m=20$ mountain ridges of variable length. Each simulated world also has $n=10$ low fertility or harsh areas as well. The low fertility areas are circular areas set such that their centre is located on one of the mountain ridges. At the centre of these areas the harshness value is 1 and it is decreasing proportionally with the square of distance from the centre. The radius of low fertility areas is set randomly between $d_{0}=50$ and $d_{\max }=100$. If a location belongs to multiple low fertility areas the harshness value of the location is the sum of the harshness values implied by each low fertility area to which the location belongs, capped at the maximum value of 1 . Figure 1 shows an example of the simulated world with mountain ridges and low fertility areas.

Each simulation starts with a random number of simulated human populations, such that a proportion around $\chi=1.5 \%$ of all spatial locations have exactly one distinct human population on them, while all others are empty at the start, i.e. the number of simulated populations in our simulations is around 90 at the beginning.

Each population is characterized by a set of abstract cultural and technical features represented by an ability string $A$ of 0 -s and $1-\mathrm{s}$, where the 1 -s indicate the presence of a such feature. Here we chose the length of ability strings to be $L=$ 100. The ability string determines the resource utilization efficiency of the population. If $A=\left(a_{1}, \ldots, a_{L}\right)$ is the ability string of a population then the resource utilization efficiency of this population is

$$
r=\Sigma_{k=1, L 1} \Sigma_{j=1, L 2} a_{L 1(k-l)+j} \cdot 2^{j}
$$

where $L 1 \cdot L 2=L$ and in our case $L 1=L 2=10$. This definition of resource utilization efficiency allows multiple cultural and technical features to contribute equally to the efficiency of the population, which is the case of real world human populations.

Figure 1. Distribution of land fertility in a simulated world. Brighter locations indicate harsh, low fertility land, darker locations indicate less harsh, more fertile land.
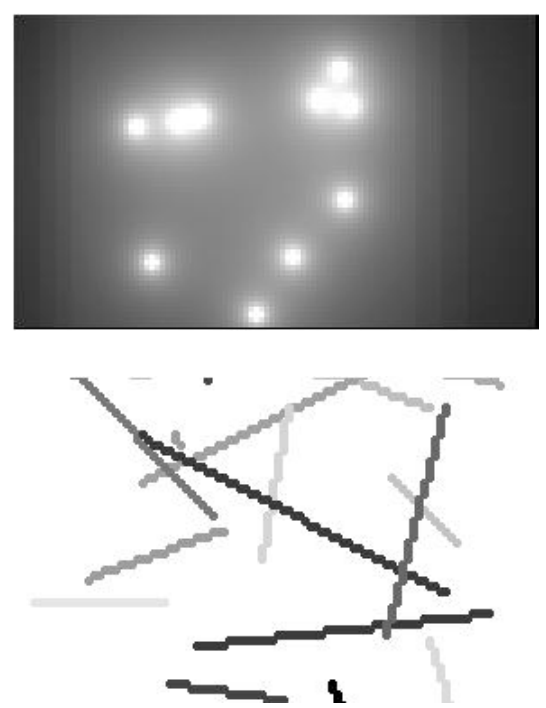

Figure 2. Mountain ridges in a simulated world. The darkness indicates the height of the ridge, darker locations are higher.

Each population may be spread over a number of space locations and each space location may house representatives from at most $N_{\max }$ (in our case this is 8 ) populations. The resource availability at each location is limited and is characterized by the harshness value of the location. Each population at each location have a basic death rate $\theta$ which is set in our case to 0.005. Each location has a maximum population size limit for each population residing in that spatial location and this depends on the resource utilization efficiency of the population and the harshness of the land at this location. The maximum size of a population with resource utilization efficiency $r$ at a location $(x, y)$ with harshness $h(x, y)$ is assumed to increase logarithmically with $r$ and to be inversely proportional with the harshness and it is set to be

$$
p_{\max }=(10+\ln (1+r)) / h(x, y)
$$

The populations at a given space location compete for the use of resources. The growth rate of the population at a space location is determined by the resource utilization efficiency of the population, the land fertility of the location, and the competition with other populations in terms of resource utilization efficiency. The growth rates of the populations are assumed to grow logarithmically with the resource utilization efficiency, to be inversely proportional with the harshness of the area and to be proportional with the level of competitiveness of the population. The death rate is considered as a subtracted element in the growth rate calculation. Assuming that there are $N$ different populations at a given spatial location $(x, y)$, and $r_{i}, i=1, \ldots, N$ are the resource utilization efficiency values for these populations the growth rates of populations are calculated as

$$
\rho_{i}(x, y)=\gamma \cdot\left(1+0.1 \cdot \ln \left(1+r_{i}\right)\right) \cdot \eta_{i} / h(x, y)-\theta
$$

where $h(x, y)$ is the harshness value at location $(x, y), \eta_{i}$ are the competitiveness factors, $\theta$ is the basic death rate. The competitiveness factor for each population depends on the ratio between the resource utilization efficiency of the considered population and the sum of the resource utilization efficiencies of all present populations and follows a sigmoidal 
curve as a function of this ratio, i.e. saturating both for very and high and very low values of the this ratio. For this simulation $\eta_{i}$ are calculated as

$$
\eta_{i}=1 /\left(1+\exp \left(\alpha \cdot\left(0.5-r_{i} /\left(\Sigma_{k=1, N} r_{k}\right)\right)\right)\right.
$$

$\gamma$ and $\alpha$ are parameters characterizing the default population growth and the level of competition - our choices were $\gamma=1.4$ and $\alpha=10$. Higher values of $\gamma$ imply quicker default population growth, higher values of $\alpha$ imply more competition between the populations.

If the size of a population at a given location grows beyond the allowed maximum size for the population at this location, the population size gets capped at the maximum allowed size. If the population size goes below zero the population disappears from this location. If the number of population at a location goes above the maximum limit for the number of different populations at a given location, the population with the smallest size gets removed.

The simulated human populations generate cultural / technical innovations randomly with $\varepsilon=0.008 \%$ chance in each time turn of the simulation - we assume that there is no cost associated with the development of these innovations. These innovations are implemented as a random flipping of cultural / technical feature indicator in the ability string $A$ from 0 to 1 or reverse. This naturally changes the resource utilization efficiency of the newly generated descendant population in comparison with the resource utilization efficiency of the parent population. A proportion of the parent population converts to the descendant population and this proportion is determined by the two resource utilization efficiency values in a similar manner that we used above for the calculation of the competitiveness of populations. If $r_{p}$ and $r_{d}$ are these values for the parent and descendent populations first we determine

$$
\eta_{p / d}=1 /\left(1+\exp \left(\alpha \cdot\left(0.5-r_{p / d} /\left(r_{p}+r_{d}\right)\right)\right)\right.
$$

then the ratio of the population converted to the new population is

$$
w=\eta_{d} /\left(\eta_{p}+\eta_{d}\right)
$$

The populations spread across the simulated world by moving from one space location to neighboring space locations, i.e. through a diffusion process. The spreading from one location to a neighboring location depends on the height value of the location from which the spreading may occur. Let us consider $Q_{i}(x, y, t)$ the number of individuals belonging to population $i$ at location $(x, y)$ at time turn $t$, the equation for the update of $Q_{i}(x, y, t)$ is the following

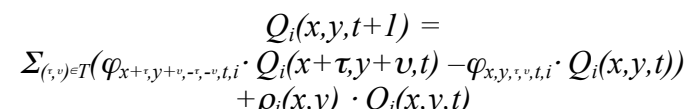

where $T=\{(-1,0),(1,0),(0,-1),(0,1)\}, \varphi_{x+r, y+v,-r,-v, t, i}$ and $\varphi_{x, y, r, v, t, i}$ are stochastic diffusivity parameters, and $\rho_{i}(x, y)$ is the growth rate calculated according to equation (4). The stochastic diffusivity parameters are set as follows

$$
\begin{gathered}
\varphi_{x+, y+y,-,-v, t, i}=\psi \quad \text { if } \\
Q_{i}(x, y, t)>\max \{g(x+\tau, y+v), g(x, y)\} \\
\text { otherwise } \\
\varphi_{x+r, y+v,-\tau,-v, t, i}=0
\end{gathered}
$$

where $g(x+\tau, y+v)$ is the height value at location $x+\tau, y+v$, with $(\tau, v) \in T \cup\{(0,0)\}$ and $\psi$ is a random value from the interval $[0, \omega]$ with $\omega<1$ (in the simulations we used $\omega=0.4$ ). This setting means that the populations can spread into the neighboring spatial locations either to the left or right or to up or down relative to the current location of the population and in any of these directions spreading happens only if the population is sufficiently large in comparison with the height of the current and neighboring locations. Note that the $\omega$ parameter influences the speed of diffusion (or diffusivity) of the populations, larger $\omega$ implying quicker spreading of the populations.

As described above the simulation starts with a number of distinct populations (around 90), which spread around in the simulated world. Populations compete with each other for the use of resources, the available amount of which is determined by the level of harshness of the spatial location (the opposite of land fertility). The spreading of populations is constrained by the presence of simulated mountain ridges. Spreading across such mountain ridges is difficult and the difficulty rises with the height of the simulated mountains. The populations randomly spawn new populations which are characterized by a cultural - technical innovation that changes their resource utilization efficiency compared to the parent population. In general these innovations may increase or may decrease the resource utilization efficiency. The simulated world evolves through a large number of time turns (we used 30,000 time turns for each simulation).

The simulations are analyzed in order to determine the dominant populations. The dominance of a population is given by the size of the population across all spatial locations relative to the size of all populations across all spatial locations of the simulated world, i.e.

$$
\pi_{i}(t)=\left(\Sigma_{(x, y)} Q_{i}(x, y, t)\right) /\left(\Sigma_{i} \Sigma_{(x, y)} Q_{i}(x, y, t)\right)
$$

All populations for which $\pi_{i}(t)>\pi_{0}=0.005$ are considered sufficiently dominant populations and considered for further analysis, i.e. these populations represent at least half percent of the total inhabitants of the simulated world.

We aim to test three hypotheses based on the observation of spreading of animal and human populations in the context of environmental constraints.

Hypothesis 1: Being close to mountain ridges offers relative protection from invasion by other populations and allows the relatively frequent development of innovations with positive effect on the resource utilization efficiency in populations residing in such spatial locations. It is expected that the closeness to simulated mountain ridges in general allows the relatively long survival of innovating populations with both useful and harmful innovations. Out of these populations the ones with useful innovations are expected to have sufficient time to grow and then expand elsewhere due to the protected nature of their origin location. 
Hypothesis 2: Existence at locations with low land fertility (harsh locations) facilitates the development of significant positive innovations. It is expected that if innovations occur in populations living in harsh conditions these innovations must be considerably positively useful in order for the innovating population to survive. Innovating populations that have harmful innovations from the perspective of their resource utilization efficiency are expected to die out quickly in the harsh environment. In such environment it is also expected that populations with useful innovations quickly outcompete populations which have lower resource utilization efficiency.

Hypothesis 3: The most dominant populations originate from locations with high land fertility (low harshness). It is expected that populations expand most quickly in high land fertility environments and developing useful innovations in such environments equips already large populations with the useful innovations which spread even more extensively than their parent population. On the other side it is also expected that populations that gain dominance on in high fertility environments must have had an earlier time when they benefited from the development of successive innovations relatively unperturbed by invaders, in line with Hypothesis 1 .

To test these hypotheses we track all populations and select for further analysis those that are sufficiently dominant (see equation (10)). For the selected populations we consider their location of origin and the location of origin of their ancestors. For these locations we consider the harshness of the location and the distance of the location from the closest simulated mountain ridge. Considering the populations along their existence we measure the length of existence of the population (population persistence time), the peak share of the population out of all inhabitants of the simulated world, and the value of the innovation that sets the population apart from its immediate ancestor populations (i.e. the difference between the resource utilization efficiency of the two populations). Following the simulation we analyze the relationships between the ridge distance and harshness of the locations of origin of the considered populations and the measured features of the populations. The analysis is repeated for multiple $(10-20)$ simulations to gather large volume of data. We also varied the values of $\gamma$ and $\alpha$ which characterize the default population growth and the level of competition among populations residing at the same spatial location in order to assess the impact of these parameters on the evolution of the simulated populations.

\section{Results and Discussion}

Each simulation was run for 30,000 time turns. The simulation parameters were set as indicated in the description of the simulations in the previous section. Each population was identified by an identification number. For each time turn we recorded the details of all extant populations, including their location of origin, the identification number of their immediate ancestor population, the ability string of the

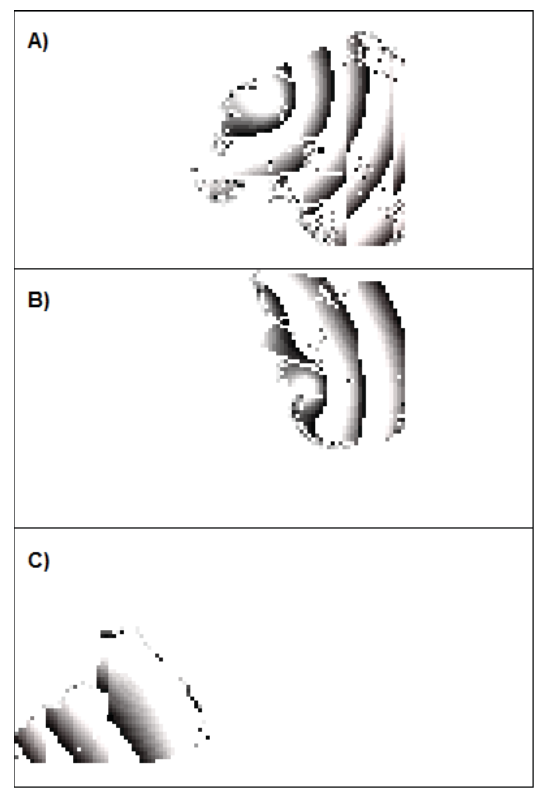

Figure 3. Turing patterns of population spreading in the simulated worlds for different values of the diffusion parameter $\omega$ : A) $\omega=0.9$ - fast spreading; B) $\omega=0.4$ - medium speed spreading; C) $\omega=0.03$ - slow spreading. White indicates no presence of the considered population, the level of darkness indicates the extent of population presence, darker meaning the presence of higher number of individuals belonging to the considered population.

population, the total size of the population across all spatial locations and the share of the population out of the total number of inhabitants in the simulated world at the given time step. The primary analysis of the simulation data was performed as described in the previous section in order to gather the information relevant for the testing of the hypotheses.

The simulations show that patterns of spreading of populations follow Turing patterning. Varying the diffusion speed parameter $\omega$ we can vary the wavelength of the populations follow Turing patterning. Varying the diffusion speed parameter $\omega$ we can vary the wavelength of the population spreading patterns, which according to the theory should be proportional with the square root of the local diffusivity value (Ouyang et al, 1995). Note that given the presence of simulated mountain ridges and low land fertility areas the diffusivity of the populations varies across the simulated world. Figure 3 shows examples of spreading patterns of populations for different settings of the diffusion speed parameter $\omega$. Similar Turing patterns have been observed in the context of patterns of spreading of animals. In the case of human populations it is less clear the evidence for such patterns. However during fast migratory periods such patterns might have emerged, for example consider the cases of two (or more) Hungarian and Bulgarian states in the middle ages, one in the area of the current countries and another (possibly more than one) in the area what is today Eastern Ukraine and South Russia (the latter fell apart during the Mongol invasion) (Tomory et al, 2007; Johanson, 2000). 

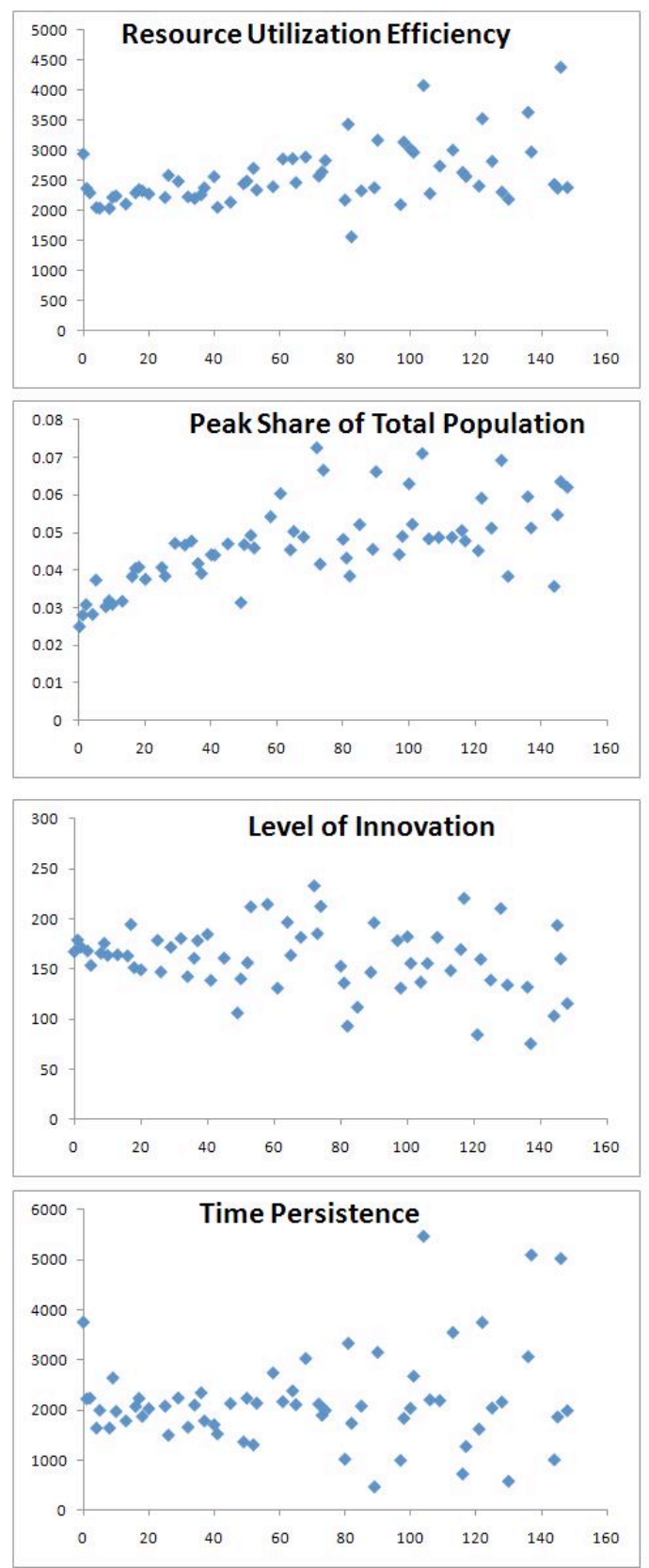

Figure 4. The relationships between the ridge distance of the location of origin of populations and the resource utilization efficiency, peak share of total population, level of innovation, and time persistence of simulated populations. The horizontal axis in all cases is the ridge distance of the population origin.

We analyzed the relationship between the distance of the location of origin of populations from the closest simulated mountain ridge (i.e. ridge distance) and the measured population performance indicators. For this purpose we pooled data from 20 simulations and calculated the average population persistence time, peak population share, resource utilization efficiency, and innovation value for each value of the ridge distance for which we found with an origin location having this value of ridge distance. The further analysis was done by considering only those ridge distance values for which we found at least 12 populations across all simulations.

We found that there is significant positive correlation $(p<0.05)$ between the ridge distance and the average resource utilization efficiency $(c=0.4926)$ and the peak share of the total population $(c=0.6263)$. We found significantly negative correlation $(c=-0.2793 ; p<0.05)$ between the ridge distance and the level of innovation (i.e. difference in resource utilization efficiency compared to the immediate ancestor population). Finally we found no significant correlation between the ridge distance and the persistence time of the population. The data is shown in Figure 4 - error bars are not shown to avoid cluttering of the figures, the error bars are in general around $10-30 \%$ of the average values.

These results indicate that Hypothesis 1 is confirmed in the sense that being close to simulated mountain ridges has a positive impact on the level of innovation in terms of resource utilization efficiency by newly emerging populations. The data does not show whether this effect is due to the protection against invasion by other populations by the mountain ridges, which are expected to slow down the spreading of populations. The results also show that populations with higher resource utilization efficiency and higher peak share of the total population emerge further away from mountain ridges.

To analyze the relationship between land fertility of the location of origin of populations and the population performance indicators we pooled the data from 20 simulations. We calculated the average performance indicators for all values of land fertility that we found and considered for further analysis those averages that were calculated from at least 12 instances of populations. The data are presented in Figure 5.

We found that there is a significant $(p<0.05)$ positive correlation between the harshness value (the opposite of land fertility) of the location of origin and the level of innovation of populations $(c=0.4146$ - note however the wavy nature of the relationship) and a significant negative correlation between the harshness value and the population's peak share of total population $(c=-0.9275)$. These two results confirm the validity of Hypotheses 2 and 3. The first result shows that indeed, the likelihood of high positive innovation increases with the harshness (low land fertility) of the location of origin of the population among populations that become sufficiently dominant. The second result shows that higher land fertility (lower harshness) of the location of origin of the population implies higher chance for the population to become highly dominant. This analysis of the data does not provide the definite explanation for these results, but the data allows further analysis, which may provide support for the hypothetical explanations that we provided in the previous section.

In terms of the relationship of the harness of the location of origin and the resource utilization efficiency and the time persistence of the population we found an unexpected relationship with three peaks at a two lower and a higher midlevel harshness value. The triple peak nature of the relationship also applies to the level of innovation as well.

To understand better the finding about the relationship of the harshness of the location of origin and the resource utilization efficiency and the time persistence of the 

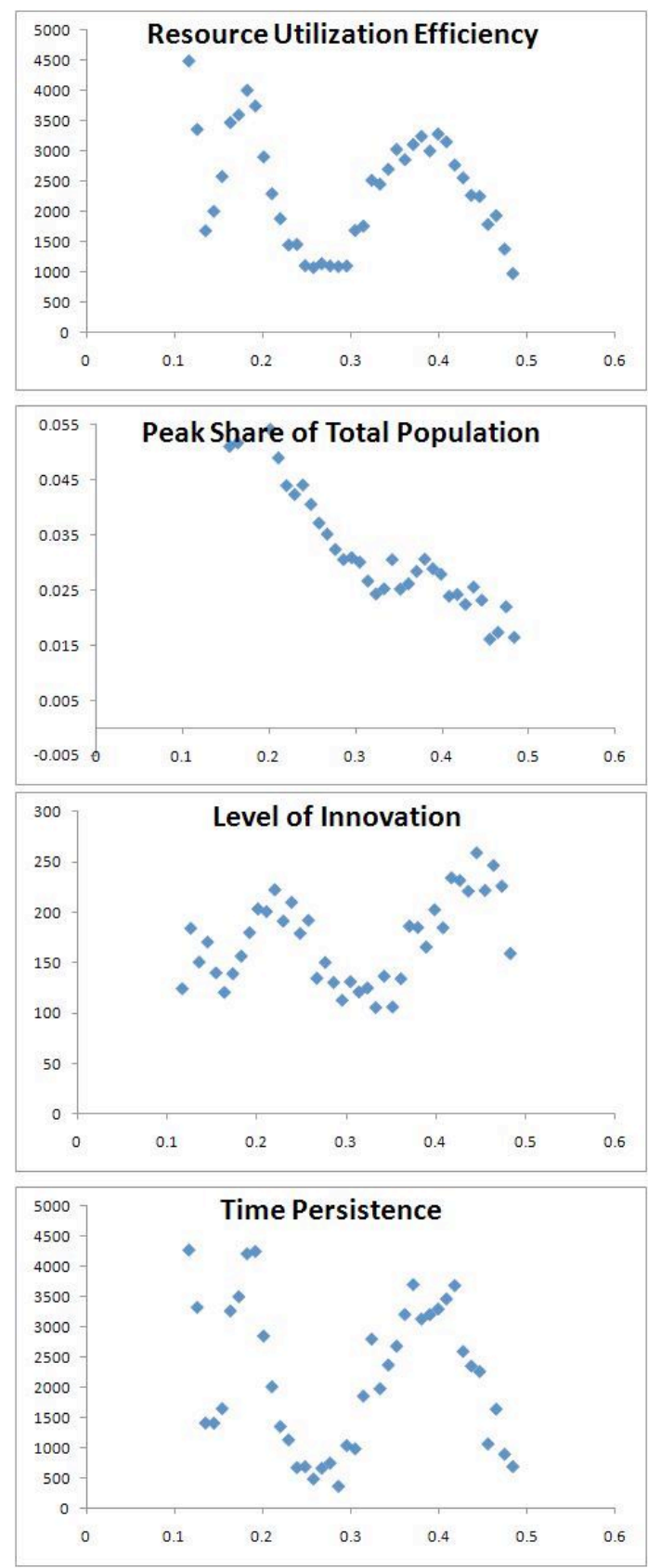

Figure 5. The relationships between the harshness of the location of origin of populations and the resource utilization efficiency, peak share of total population, level of innovation, and time persistence of simulated populations. The horizontal axis in all cases is the harshness of the population origin.

population we varied the values of the parameters $\gamma$ and $\alpha$, which characterize the default population growth and the level of competition among populations residing at the same spatial location. We considered $\gamma$ values in the range of 0.8 to 1.7 , and $\alpha$ values in the range of 6 to 12 . We found that the behavior of these two relationships shows a wave-like nature with a number of peaks in general - see Figures 6 and 7. Note that these waves are not spatial waves in the simulated world,

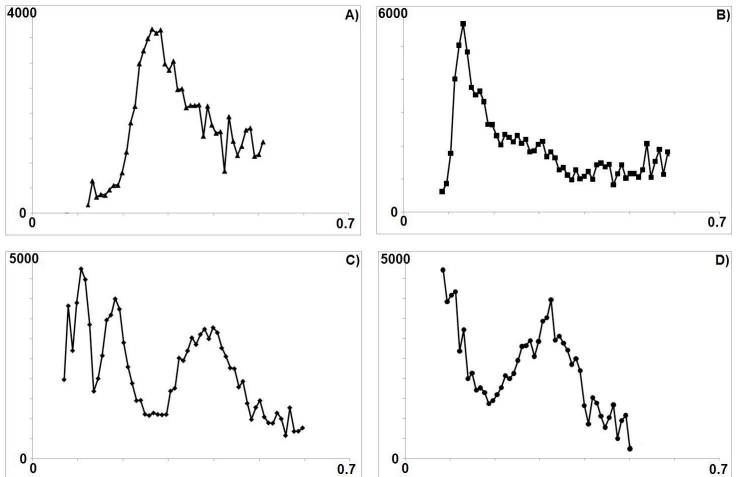

Figure 6. The average resource utilization efficiency of sufficiently dominant populations as function of the harshness of the location of origin - A) $\alpha=6$; B) $\alpha=8$; C) $\alpha=10$; D) $\alpha=12$; the lines connect the data points.

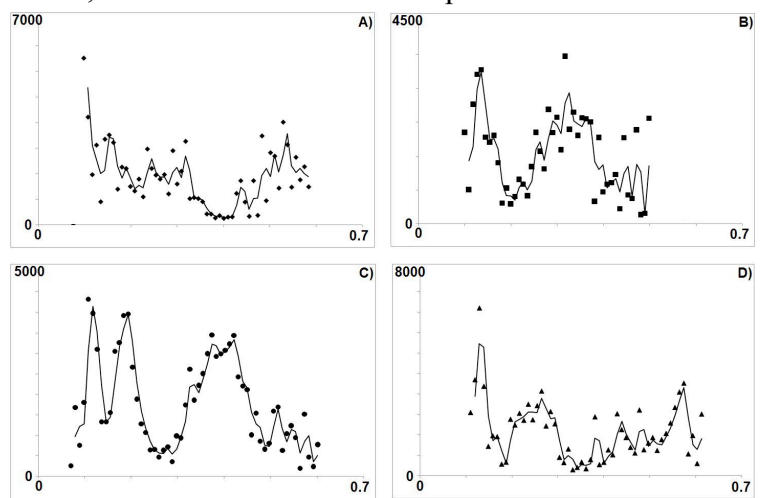

Figure 7. The average time persistence of sufficiently dominant populations as function of the harshness of the location of origin - A) $\gamma=0.8$; B) $\gamma=1.1$; C) $\gamma=1.4$; D) $\gamma=1.7$; the lines are the moving averages.

but waves depending on the harshness of the location of origin of the population.

Considering equation (1) that describes the general process of population spreading, the result about the wave-like dependence of the resource utilization efficiency and time persistence on the harshness of the location of origin suggests that reaction term of equation $(1)-F(u, x, t)$, which depends on the land fertility of the location, plays an important role in determining which locations are more likely to give birth to the longest existing and most resource efficient populations These locations are consequently relatively over-represented among the locations of origins of populations that are sufficiently dominant. A possible explanation is that there is some interference between the wave like nature of population spreading (see the Turing patterns above in Figure 3) and the land fertility dependent reaction term of the population spreading equation, which constrains location of origin of the most resource efficient and longest existing populations to certain bands of land fertility values and locations which have such land fertility.

The implication of this result is that it is possible that for real human, animal, and plant populations as well, the locations of origins of most populations may not be the most fertile and supportive lands, but rather places with intermediate level of land fertility (or environmental 
supportiveness in general in the case of animals and plants), which match in some sense the wavelength of the waves of spreading of these populations.

\section{Conclusions}

In this paper we investigate the role of environmental factors in the emergence of cultural and technical innovations in human populations through simulation based analysis. We did not consider the possibility of copying of innovations by colocated populations and neither the possible impact of available excess resources. We used a relatively simple simulated world with simulated mountain ridges and low fertility land areas to analyze the impact of these factors on the successful spreading and survival of simulated human populations. The results show that both environmental factors matter for the emergence of successful populations.

Our results show that in the simulated world being close to mountain ridges correlates with the development of innovations with significant positive effect on the resource utilization efficiency in populations originating from such spatial locations. This matches well with the observation that several highland areas with mountain protected valleys facilitate the existence of many distinct human populations, each developing different cultural and technical innovations (Bulayeva et al, 2003; Reesink et al, 2009). Our simulations suggest that such areas are likely to lead to the emergence of cultural and technical innovations that give a significant competitive advantage to a local human population in comparison with neighboring populations and these populations are likely to spread out form these valleys and may become dominant populations in larger areas.

The results show that populations originating from locations with low land fertility are likely to develop significant positive innovations. The underlying reason may be that other populations originating from such areas that do not develop such significant positive cultural and technical innovations simply do not survive. The implication of this finding is that it can be expected that human populations with the most beneficial cultural and technical innovations may originate from areas that are particularly challenging environments.

Finally, we found that the most dominant simulated human populations originate from locations with high land fertility within the simulated worlds. This finding matches well with the evidence that shows that historically many of the most successful human populations get established in high land fertility areas (e.g. the fertile alluvial valleys along large rivers such as the Nile, Tigris, Euphrates, Huang He, Yangtze) (Fukuyama, 2014).

The three key findings in combination suggest that possibly the most successful human populations may have roots in harsh lands, possibly protected by mountain ridges, where their ancestors developed key cultural and technical innovations. The descendants of these ancestral populations may become really successful following migration and arrival to fertile land areas where they can expand and take full benefit of their competitive advantage relative to other populations, which is due to their cultural and technical innovations.

\section{References}

Barraclough, T.G., Vogler, A.P., Harvey, P.H. (1998). Revealing factors that promote speciation. Philosophical Transactions of the Royal Society of London B, 353: 241-249.

Bouckaert, R., Lemet, P., Dunn, M., Greenhill, S.J., Alekseyenko, A.V., Drummond, A.J., Gray, R.D., Suchard, M.A., Atkinson, Q.D. (2012). Mapping the origins and expansion of the Indo-European language family. Science, 337: 957-960.

Bulayeva, K., Jorde, L.B., Ostler, C., Watkins, S., Bulayev, O., Harpending, H. (2003). Genetics and population history of Caucasus populations. Human Biology, 75(6): 837-853.

Cheng, H., Yao, N., Huang, Z.-G., Park, J., Do, Y., Lai, Y.-C. (2014). Mesoscopic interactions and species coexistence in evolutionary game dynamics of cyclic competitions. Scientific Reports, 4: 7486.

Chiaroni, J., King, R.J., Underhill, P.A. (2008). Correlation of annual precipitation with human Y-chromosome diversity and the emergence of Neolithic agricultural and pastoral economies in the Fertile Crescent. Antiquity, 82: 281-289.

Delre, S.A., Jager, W., Bijmolt, T.H.A., Janssen, M.A. (2010). Will it spread of not? The effects of social influences and network topology on innovation diffusion. Journal of Product Innovation Management, 27: 267-282.

Der Sarkissian, C. et al (2013). Ancient DNA reveals prehistoric geneflow from Siberia in the complex human population history of North East Europe. PLoS Genetics, 9(2): e1003296.

Diamond, J. (1997). Guns, Germs, and Steel: The Fates of Human Societies. Norton, New York.

Diamond, J., Bellwood, P. (2003). Farmers and their languages: The first expansions. Science, 300:597-603.

Fukuyama, F. (2014). Political Order and Political Decay. Profile Books, London.

Garcia-Ramos, G., Rodriguez, D. (2001). Evolutionary speed of species invasions. Evolution, 56(4): 661-668.

Jeltsch, F., Muller, M.S., Grimm, V., Wissel, C., Brandl, R. (1997). Pattern formation triggered by rare events: lessons from the spread of rabies. Proceedings of the Royal Society of London B, 264: 495503.

Johanson, L. (2000). Linguistic convergence in the Volga area. Studies in Slavic and General Linguistics, 28: 165-178.

Lambin, X., Elston, D.A., Petty, S.J., MacKinnon, J.L. (1998). Spatial asynchrony and periodic travelling waves in cyclic populations of field voles. Proceedings of the Royal Society of London B, 265: 1491-1496.

Macy, M.W., Willer, R. (2002). From factors to actors: Computational sociology and agent-based modeling. Annual Review of Sociology, 28: 143-166.

Mahajan, V., Muller, E. (1979). Innovation diffusion and new product growth models in marketing. Journal of Marketing, 43(4): 55-68.

Mellars, P. (2006). Why did modern human populations disperse from Africa ca. 60,000 years ago? A new model. PNAS, 103(25):93819386.

Ouyang, Q., Li, R., Li, G., Swinney, H.L. (1995). Dependence of Turing pattern wavelength on diffusion rate. Journal of Chemical Physics, 102(6): 2551-2555.

Petrovskii, S.V., Morozov, A.Y., Venturino, E. (2002). Allee effect makes possible patchy invasion in a predator-prey system. Ecology Letters, 5: 345-352.

Reesink, G., Singer, R., Dunn, M. (2009). Explaining the linguistic diversity o Sahul using population models. PLoS Biology, 7(11): e1000241.

Tomory, G., Csanyi, B., Bogacsi-Szabo, E., Kalmar, T., Czibula, A., Csosz, A., Priskin, K., Mende, B., Lango, P., Downes, C.S., Rasko, I. (2007). Comparison of maternal lineage and biogeographic analyses of ancient and modern Hungarian populations. American Journal of Physical Anthropology, 134: 345-368.

Warren, D.L., Glor, R.E., Turelli, M. (2008). Environmental niche equivalency versus conservatism: quantitative approaches to niche evolution. Evolution, 62(11): 2868-2883. 